BMJ Nutrition,

Prevention \& Health

\title{
Temporal variations in the severity of COVID-19 illness by race and ethnicity
}

Joseph E Ebinger (D) , ${ }^{1,2}$ Matthew Driver, ${ }^{1,2}$ Hongwei Ji, ${ }^{3,4}$ Brian Claggett, ${ }^{5}$ Min Wu, ${ }^{1,2}$ Eric Luong, ${ }^{1,2}$ Nancy Sun, ${ }^{1,2}$ Patrick Botting, ${ }^{1,2}$ Elizabeth H Kim, ${ }^{1,2}$ Amy Hoang, ${ }^{1,2}$ Trevor Trung Nguyen, ${ }^{1,2}$ Jacqueline Diaz, ${ }^{1,2}$ Eunice Park, ${ }^{6}$ Tod Davis, ${ }^{6}$ Shehnaz Hussain, ${ }^{7}$ Susan Cheng, ${ }^{1,2}$ Jane C Figueiredo ${ }^{1,8,9}$

\section{ABSTRACT}

Introduction Early reports highlighted racial/ethnic disparities in the severity of COVID-19 seen across the USA; the extent to which these disparities have persisted over time remains unclear. Our research objective was to understand temporal trends in racial/ethnic variation in severity of COVID-19 illness presenting over time. Methods We conducted a retrospective cohort analysis using longitudinal data from Cedars-Sinai Medical Center, a high-volume health system in Southern California. We studied patients admitted to the hospital with COVID-19 illness from 4 March 2020 through 5 December 2020. Our primary outcome was COVID-19 severity of illness among hospitalised patients, assessed by racial/ethnic group status. We defined overall illness severity as an ordinal outcome: hospitalisation but no intensive care unit (ICU) admission; admission to the ICU but no intubation; and intubation or death

Results A total of 1584 patients with COVID-19 with available demographic and clinical data were included. Hispanic/Latinx compared with non-Hispanic white patients had higher odds of experiencing more severe illness among hospitalised patients (OR $2.28,95 \% \mathrm{Cl}$ 1.62 to 3.22 ) and this disparity persisted over time. During the initial 2 months of the pandemic, nonHispanic blacks were more likely to suffer severe illness than non-Hispanic whites (OR 2.02, 95\% Cl 1.07 to 3.78); this disparity improved by May, only to return later in the pandemic.

Conclusion In our patient sample, the severity of observed COVID-19 illness declined steadily over time, but these clinical improvements were not seen evenly across racial/ethnic groups; greater illness severity continues to be experienced among Hispanic/Latinx patients.

\section{INTRODUCTION}

The emergence and rapid spread of the novel SARS-CoV-2 have exposed and exacerbated racial and ethnic health disparities across the country. ${ }^{1-5}$ Prior analyses of disparities in COVID-19 illness have not fully accounted for variations in underlying comorbidities or assessed the extent to which severity of COVID-19 clinical presentations is evolving over the course of the pandemic. ${ }^{6-11}$ There is strong reason to suspect that severity of

\section{What this paper adds}

The COVID-19 pandemic has disproportionately affected minority communities. Datafrom early in the pandemic demonstrated worse outcomes, particularly amongAfrican Americans.

- COVID-19 severity of illness among non-Hispanic blacks initially improved, however, but is showing signs of returning.

- Severity of COVID-19 illness among the Hispanic/ Latinx population remained higher than nonHispanic whites during most of the pandemic and shows signs of worsening.

- COVID-19 severity of illness among minority populations has changed during the course of the pandemic, but has been persistently worse among Hispanic/ Latinx patients. Growingdisparities in severity of illness may reflect decreased ability of the minority population to limit exposure to the virus, aswell as obtaining less benefit from novel treatments for COVID-19.

illness is not only changing over time but also varying by race and ethnicity—at least partly due to the dynamic interplay between public health efforts to limit disease spread, improvements in medical therapies, and racial/ethnic clustering of socioeconomic factors such as essential employment status, neighbourhood infrastructure and trust in the medical system. ${ }^{12}$ To better understand racial/ethnic variation in how COVID-19 illness severity is changing over time, while accounting for the effects of pre-existing conditions and socioeconomic factors, we conducted multivariable-adjusted temporal trends analyses using longitudinal data collected from our healthcare system, which serves as a primary high-volume clinical centre for treating COVID-19 illness affecting the diverse urban population of the greater Los Angeles County. 


\section{METHODS}

\section{Study population}

In this retrospective cohort study, we identified all patients admitted to the hospital with confirmed SARS-CoV-2 infection in our large multisite health system based in Los Angeles, California (Cedars-Sinai Medical Center, CSMC), from 4 March through 5 December 2020. As the largest non-profit hospital in western USA, CSMC serves a catchment area of $>1.8$ million people, with over a quarter million inpatient hospital days for admitted patients, $>90$ 000 emergency department visits and nearly 800000 outpatient appointments each year. All laboratory testing for SARS-CoV-2 were performed using reverse transcriptase PCR of extracted RNA from nasopharyngeal swabs at the Los Angeles Department of Public Health from 4 March to 21 March 2020 and thereafter at the CSMC Department of Pathology and Laboratory Medicine. A limited number of patients $(1.0 \%)$ received SARS-CoV-2 testing at an outside facility, and documentation of a positive test result was reviewed by medical staff and considered comparable for accuracy.

\section{Data collection}

We curated demographic, clinical and outcomes data from the electronic health record (EHR) and confirmed all key variables with manual adjudication. We defined race and ethnicity membership as follows: Hispanic/ Latinx ethnicity (all races), non-Hispanic black (NHB), Asian/Pacific Islander, non-Hispanic white (NHW) and other (including individuals with multiple races listed). We used data from the American Community Survey 2019 to calculate neighbourhood socioeconomic status (SES) for each patient census tract based on the Agency for Healthcare Research and Quality (AHRQ) SES index, ${ }^{13}$ which includes measures of unemployment, household income, property value, poverty level and educational attainment. We mapped patients to a census tract using their primary address listed in the EHR.

To capture relative comorbid status at the time of presentation to medical care, the Elixhauser Comorbidity Index (ECI) score, using van Walraven weighting, ${ }^{14}$ was calculated for all patients based on the International Classification of Diseases-10 codes present prior to or at the time of initial presentation for COVID-19. Hospitalisation characteristics including length of stay, admission to an intensive care unit (ICU), intubation and in-hospital death were ascertained from time stamps recorded for admission, unit transfers and discharge. Interventions such as intubation were identified through time-stamped orders in the EHR and verified by manual chart review.

\section{Clinical outcomes}

Our primary objective was to evaluate temporal trends of COVID-19 severity of illness among hospitalised patients by race and ethnicity. Hospitalised patients, rather than all patients diagnosed with COVID-19, were selected given the known variation in rates of presentation to care over time, particularly in relation to periodically imposed lockdowns that may have influenced patterns of detection and diagnosis of the milder forms of COVID-19 illness. We defined severity of illness as an ordinal variable, based on a modified core outcome measure scale ${ }^{15}: 0=$ required hospitalisation but no ICU or intubation (least severe); $1=$ required hospitalisation and ICU but no intubation; and $2=$ required hospitalisation, ICU, and intubation or death (most severe).

\section{Statistical analysis}

We summarised demographic and clinical characteristics using mean and SD for continuous variables and counts with percentages for categorical variables. We used $\chi^{2}$ test and one-way analysis of variance to compare demographic and clinical characteristics, by racial/ ethnic subgroups, within four discrete time periods. Time periods were calculated based on quartiles of the cumulative distribution of daily COVID-19 admissions (in our sample) to account for variations in incidence over the course of the pandemic: period 1, from 4 March to 7 May; period 2, from 8 May to 14 July; period 3, from 15 July to 20 October; and period 4, from 21 October to 5 December. We conducted multivariate ordinal logistic regression to examine the association between race/ ethnicity and severity of illness adjusting for age, sex, ECI and SES index both overall and for each distinct time period. We fit models using restricted cubic splines with seven knots to account for dynamic non-linear temporal trends in illness severity observed over the course of the study period for all patients as well as among hospitalised patients. Racial/ethnic differences in temporal trends in illness severity were evaluated using likelihood ratio tests between models with and without interaction terms between race/ethnicity and the cubic spline variable representing time. To further evaluate severity of illness at different time points throughout the study period, we also fit models stratified by time period. Racial/ethnic differences in illness severity across the four discrete time periods were evaluated using joint hypothesis testing. All analyses were conducted using R V.3.4.1 (R Foundation for Statistical Computing, Vienna, Austria) and $p$ values $<0.05$ were considered significant.

\section{Patient and public involvement}

Patients and the public were not involved in the development of this study.

\section{RESULTS}

\section{Cohort characteristics}

We identified a total of 1636 COVID-19-positive patients admitted to the hospital, of whom $51(3.1 \%)$ did not have documented race or ethnicity and 1 did not have a documented date of birth and were thus excluded from analyses. For the final sample, including 1584 patients, the mean age was $61.6 \pm 19.8$ years, $874(55.2 \%)$ were men, the median household income was estimated at \$36 118 (IQR \$23 359-\$54207), and the mean SES index was 56.6 \pm 6.8 


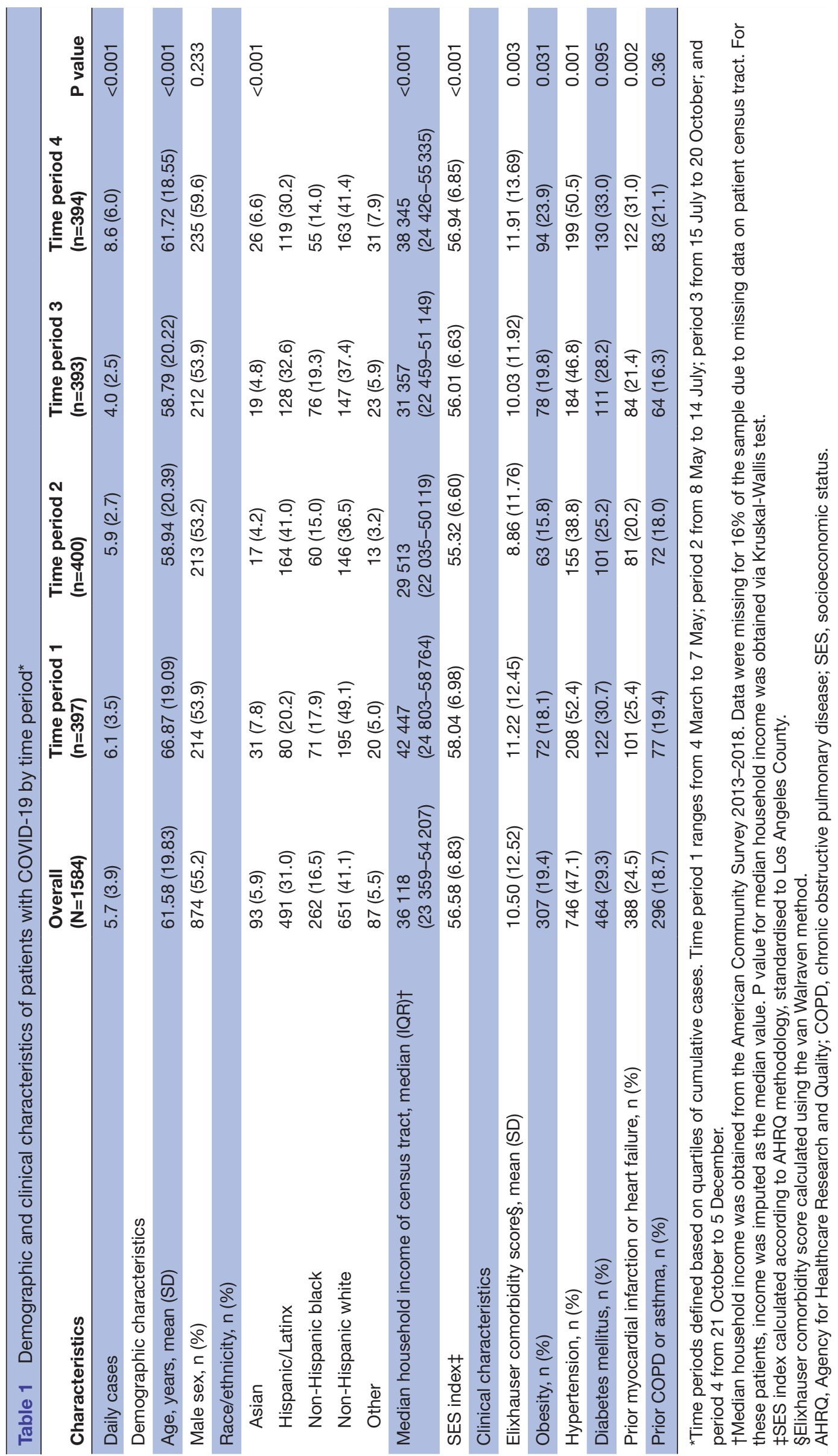




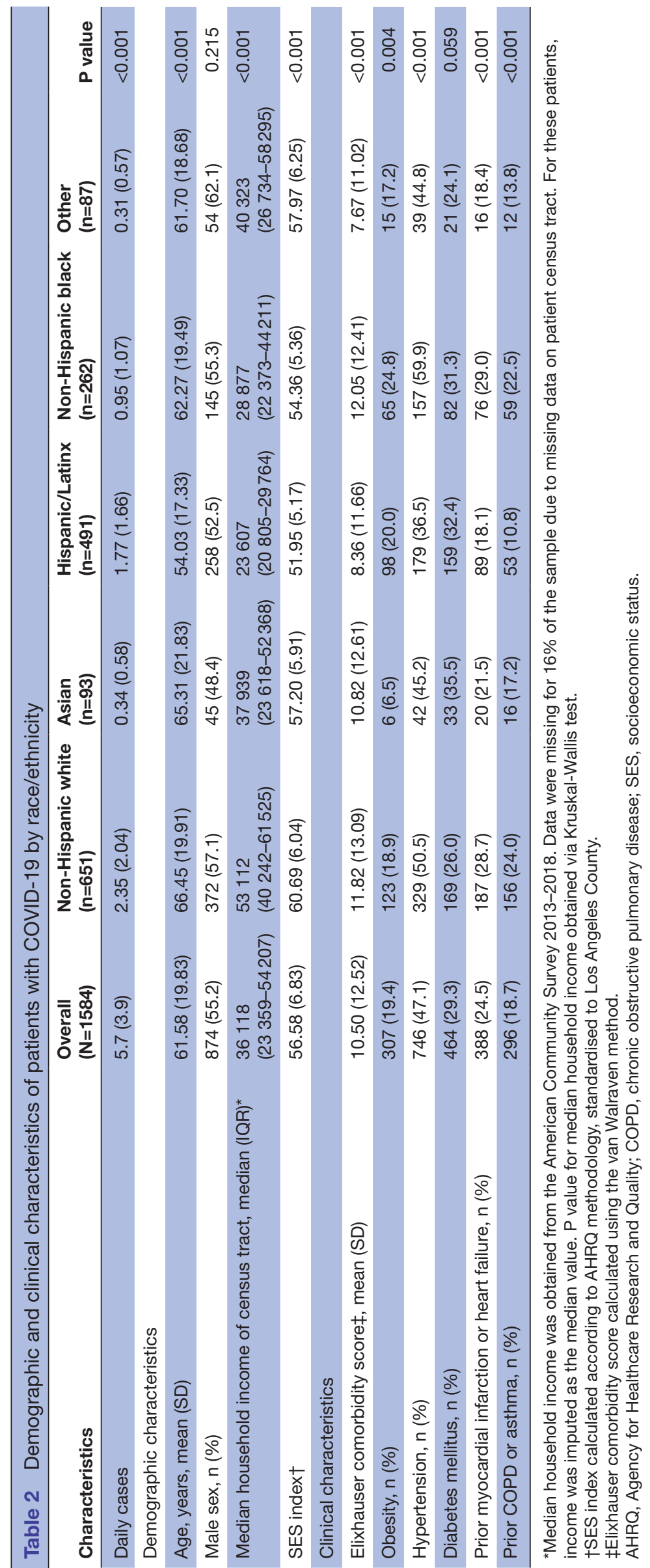



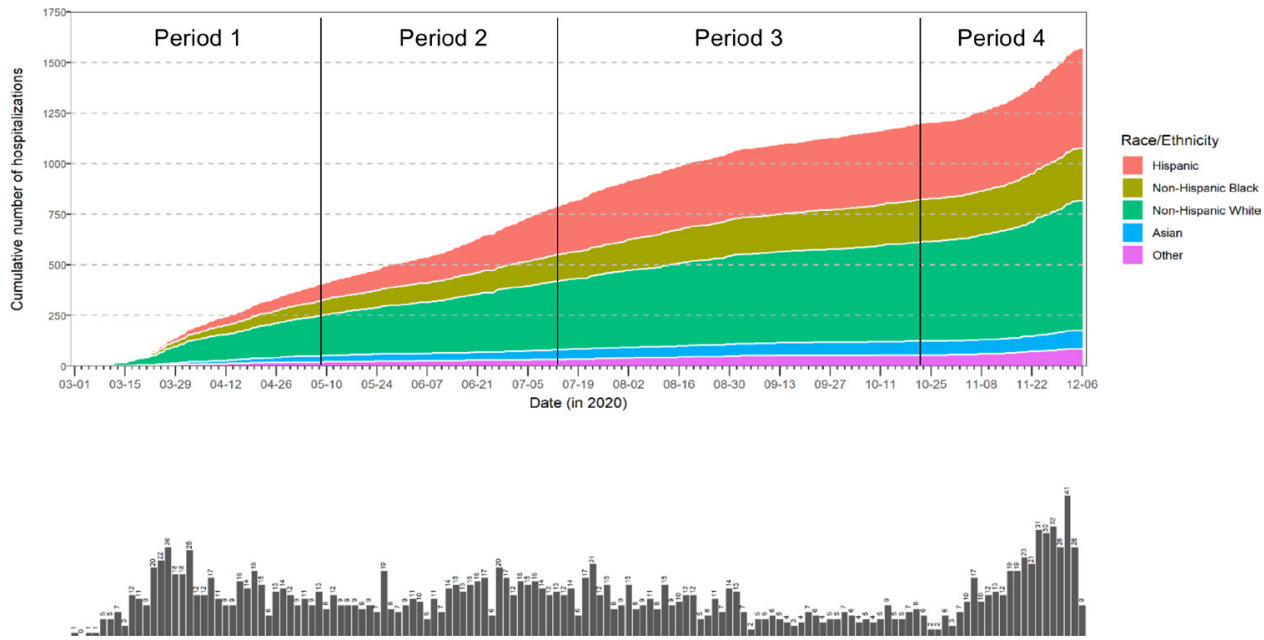

Figure 1 Cumulative COVID-19 cases over time by race/ethnicity.

(table 1). Hypertension was the most common comorbidity $(47.1 \%)$, followed by diabetes mellitus $(29.3 \%)$ and prior myocardial infarction or heart failure $(24.5 \%)$. A total of $651(41.1 \%)$ identified as NHW, $262(16.5 \%)$ as NHB, $491(31.0 \%)$ as Hispanic/Latinx, $93(5.9 \%)$ as Asian and $87(5.5 \%)$ as other race/ethnicity. Compared with NHWs, Hispanic/Latinx and NHB patients tended to be younger, have lower estimated median income and higher rates of diabetes mellitus; NHBs also tended to have higher frequency of hypertension, while hypertension was less common among Hispanic/Latinx patients (table 2).

During the study period, 201 (12.7\%) patients required ICU admission without need for intubation and 156 $(9.8 \%)$ required intubation or suffered in-hospital death. The frequency of clinical outcomes, by level of illness severity, is shown by racial/ethnic group in online supplemental table 1. Among patients admitted to the hospital for whom vital sign data from the emergency department were available $(n=1551)$, we examined the first-encounter vital signs. The distribution of first-encounter vital signs overall and by racial/ethnic group is shown in online supplemental table 2 . We observed statistically significant differences across racial/ethnic groups in certain

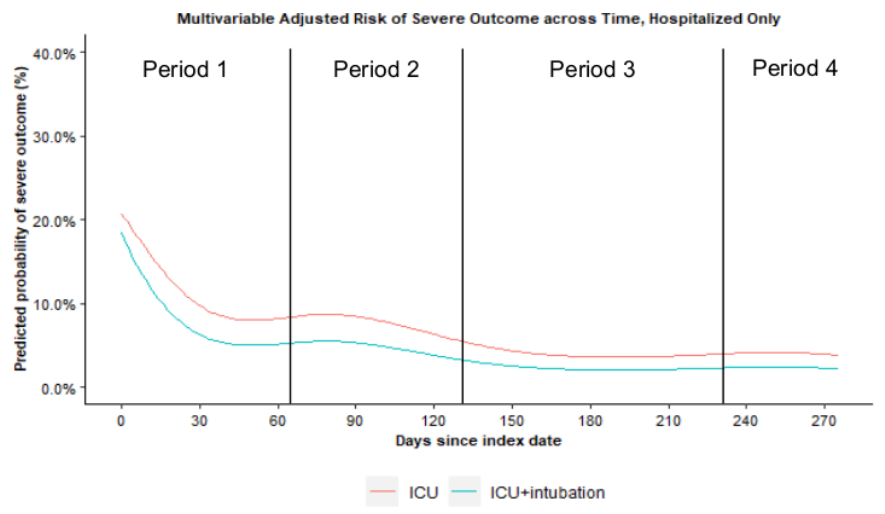

Figure 2 Predicted probability of severe COVID-19 illness over time, among hospitalised patients. ICU, intensive care unit. first-encounter vital signs, although for very narrow average ranges of difference: oxygen saturation (difference range $<1 \%)$, heart rate $(<5$ beats per minute) and systolic blood pressure $(<6 \mathrm{~mm} \mathrm{Hg})$.

\section{Crude temporal variations}

The average daily rate of new COVID-19 admissions was $5.7 \pm 3.9$ cases per day over the study period, with variations seen over time (figure 1). We observed COVID-19 admis-

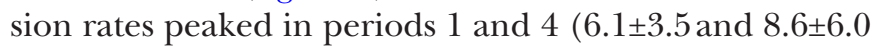
cases per day, respectively) and nadir in periods 2 and 3 $(5.9 \pm 2.7$ and $4.0 \pm 2.5$ cases per day, respectively) (table 1$)$. The average patient age was highest at the beginning of the pandemic in period $1(66.9 \pm 19.1)$, lowest in period 3 (58.8 \pm 20.2$)$ and increased again in period $4(61.7 \pm 18.6)$. Overall comorbidity status, as measured by the ECI, was

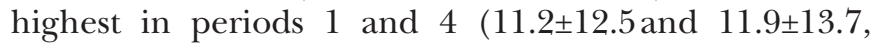

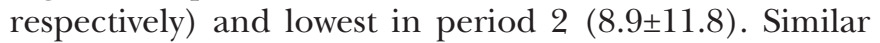
trends were observed among NHWs in analyses stratified by race/ethnicity (online supplemental table 3). NHBs did not demonstrate similar reductions in ECI during period 2 and ECI was relatively lower for Hispanic/Latinx patients throughout the study period. Rates of intubation and death steadily decreased over time, while ICU utilisation initially declined from periods 1 to 3 before increasing again during period 4 (table 2 ). Similar trends were appreciated when outcomes were stratified by race/ ethnicity.

\section{Multivariable-adjusted temporal variations by race and ethnicity}

In multivariable analysis, we observed that the risk of developing more severe illness steadily decreased over time (figure 2). When stratified by race/ethnicity, Hispanic/ Latinx patients were more likely to experience severe illness compared with NHWs (OR 2.28, 95\% CI 1.62 to 3.22). This finding was consistent across time periods, except for time period 3, although a non-significant trend towards more severe disease for Hispanic/Latinx populations was still observed. Similar to analyses of overall 


\begin{tabular}{|c|c|c|c|c|c|c|}
\hline & $\begin{array}{l}\text { Overall } \\
\text { OR }(95 \% \mathrm{Cl})\end{array}$ & $\begin{array}{l}\text { Time period } 1 \\
\text { OR }(95 \% \mathrm{Cl})\end{array}$ & $\begin{array}{l}\text { Time period } 2 \\
\text { OR }(95 \% \mathrm{Cl})\end{array}$ & $\begin{array}{l}\text { Time period } 3 \\
\text { OR }(95 \% \mathrm{Cl})\end{array}$ & $\begin{array}{l}\text { Time period } 4 \\
\text { OR }(95 \% \mathrm{Cl})\end{array}$ & $P$ value \\
\hline Non-Hispanic white & Ref & Ref & Ref & Ref & Ref & \\
\hline Hispanic/Latinx & 2.28 (1.62 to 3.22$)$ & 2.67 (1.34 to 5.34$)$ & 2.40 (1.25 to 4.69$)$ & 1.80 (0.86 to 3.87$)$ & 3.87 (1.75 to 8.86$)$ & 0.105 \\
\hline
\end{tabular}

Bold indicates a statistically significant vaue.

${ }^{*}$ Outcome of COVID-19 illness severity score in hospitalised patients was defined as an ordinal variable where $0=$ required admission but never ICU-level care; 1=required ICU-level care but never intubation; 2=required intubation. Models adjusted for race/ethnicity, van Walraven Elixhauser comorbidity score, age, census tract, SES index and sex.

†Time periods defined based on quartiles of cumulative cases. Time period 1 ranges from 4 March to 7 May; period 2 from 8 May to 14 July; period 3 from 15 July to 20 October; and period 4 from 21 October to 5 December. $P$ value represents joint test for difference in OR across time periods for each race/ethnicity category.

$\ddagger$ Asian and other race collapsed due to small sample size among hospitalised patients.

ICU, intensive care unit; Ref, reference; SES, socioeconomic status.

illness severity, NHB patients were more likely to experience more severe illness than NHWs in periods 1 and 4 (2.02, 1.07 to 3.78 ; and $2.66,1.08$ to 6.48 , respectively), without significant differences appreciated during the remainder of the study period (table 3, online supplemental figure 1).

\section{DISCUSSION}

In our high-volume academic medical centre, serving a diverse urban population that has been substantially affected by the pandemic, we found that COVID-19 illness severity steadily decreased over time. However, the temporal improvement in severity of COVID-19 illness among our patients was not seen evenly across racial/ ethnic groups. Importantly, among patients hospitalised for COVID-19, Hispanic/Latinx patients were consistently at the highest risk for the most severe forms of COVID-19 illness compared with NHWs, and this disparity not only persisted throughout the course of the pandemic but may be worsening with time. Further, improvements in disparate outcomes for NHBs seen earlier in the pandemic appear to be re-emerging.

Our findings extend from prior reports of minority populations experiencing greater severity of illness and poorer outcomes due to COVID-19. As early as March 2020, emerging studies indicated that NHBs were being hospitalised at $33 \%$ higher rates than expected across the USA. ${ }^{16}$ Data collected from subsequent single-site ${ }^{17-21}$ and regional studies nationally ${ }^{22} 23$ and globally ${ }^{24} 25$ also confirmed disproportionately worse COVID-19 outcomes being experienced by racial/ethnic minority populations. Our findings confirm that racial/ethnic disparities in COVID-19 outcomes not only exist across a large metropolitan population in Southern California, but that these disparities are persisting over time-even after accounting for variations in underlying pre-existing comorbidities that are known to influence illness susceptibility. In fact, Hispanic/Latinx patients in our sample have greater risk for more severe forms of COVID-19 illness even while consistently being younger and with fewer comorbid conditions compared with NHWs. Importantly, our data highlight an elevated risk of severe COVID-19 illness in Hispanic/Latinx patients has not only been constant over time but appears to be increasing. For most of the study period, hospitalised Hispanic/Latinx patients were more than twice as likely to require ICU admission, be intubated or die than NHW patients; now, by the end of the study period, Hispanic/Latinx are nearly four times as likely to suffer severe COVID-19 illness. By contrast, initially observed disparities for NHBs disappeared within 2-3 months into the pandemic, but are now re-emerging, with NHBs 2.7 times as likely to suffer severe COVID-19 illness during the most recent time period in our study.

There are several potential explanations for our findings. The overall decline in COVID-19 illness severity, with a reported mortality rate as high as $16.6 \%$ in JanuaryApril and declining to $9.3 \%$ by May-June, ${ }^{26}$ has been associated with broadening demographics of the populations at risk, in addition to rapidly advancing therapeutics such as remdesivir, baricitinib and monoclonal antibodies. ${ }^{27-29}$ As for other disease states, new therapeutics are often not available to all segments of society simultaneously and thus can exacerbate disparities in healthcare access, utilisation and outcomes. ${ }^{30-32}$ Beyond variations in access to therapeutics, racial/ethnic disparities in clinical outcomes can arise from the complex interplay between sociodemographic factors and intrinsic disease risk. ${ }^{33}$ For Hispanic/ Latinx compared with NHW patients in our sample, the only characteristic that consistently differed was lower SES index (driven in part by lower estimated median household income). Factors related to lower SES may have led to a greater delay in presentation with COVID-19 illness even though we did not observe clinically meaningful racial/ethnic differences in first-encounter vital signs. Unmeasured or otherwise undetected subclinical risk factors may also be driving the persistent disparities 
seen in our sample. For example, malnutrition and low levels of vitamin $\mathrm{D}$ have each been associated with adverse COVID-19 outcomes, but were unable to be adequately controlled for in this analysis. ${ }^{34}$ Notably, at least one prior study of racial/ethnic disparities in COVID-19 severity, involving over 2600 patients hospitalised in New York City during the first few months of the pandemic, paradoxically found that NHBs and Hispanic/Latinx patients had less illness severity than NHW patients. ${ }^{36}$ In accordance with other studies reporting more affluent and travelled individuals more affected earlier in the pandemic, ${ }^{37}$ followed by less affluent and more essential worker individuals increasingly affected later in the pandemic, ${ }^{33}$ severity of COVID-19 illness may be determined by relative dose, type, timing and chronicity of SARS-CoV-2 exposure on top of factors specific to race/ ethnicity and SES.

Several limitations to our study merit consideration. Our data were derived from a single healthcare system, although high-volume and serving a dense and diverse urban population. The extent to which our findings are generalisable to other populations requires further investigation. Our analyses relied on the AHRQ SES index as a surrogate for patient SES. Importantly, this variable is a marker of SES of the entire community in the census tract in which a patient lives and may not be reflective of an individual patient's own SES. While this is a wellaccepted method for controlling for SES, patient-level data (individual patient employment status, income, housing and so on) would allow for improved controlling for SES but were not readily available through medical records for the present study. Finally, race and ethnicity are complex sociocultural entities ${ }^{38}$ which often oversimplify the heterogeneity that exists within broadly defined categories. For example, more granular analyses of ethnic disparities can be pursued by examining subgroups of Hispanic/Latinx communities identified using data on ancestry, immigration status and degree of acculturation. Although the racial/ethnic categories in this analysis are widely used to examine group differences across the population, they do not represent the range of experiences, situations and other factors pertinent to the individual members of these various populations.

In summary, our study findings highlight temporal trends in the severity of COVID-19 illness that are particularly relevant to the health of racial/ethnic minorities. Specifically, while severity of illness decreased among the entire population over time, Hispanic/Latinx patients consistently experienced greater illness severity than NHWs throughout the pandemic, despite having similar hospitalisation rates and a lower burden of pre-existing risk factors. Whereas NHBs in our patient population experienced more severe illness when the pandemic first began, their clinical outcomes improved in the middle of the study period, only to re-emerge later on. Further concerning is the finding that this disparity is not only persisting but potentially even worsening over time. Future studies are needed to comprehensively determine the extent to which this Hispanic/Latinx and NHB disparity represents biological differences in susceptibility to severe COVID-19 illness or variations in the social determinants of health, or both.

\section{Author affiliations}

${ }^{1}$ Department of Cardiology, Cedars-Sinai Medical Center, Los Angeles, California, USA

${ }^{2}$ Smidt Heart Institute, Cedars-Sinai Medical Center, Los Angeles, California, USA ${ }^{3}$ Department of Cardiology, The Affiliated Hospital of Qingdao University, Qingdao, Shandong, China

${ }^{4}$ Department of Cardiology, Shanghai Tenth People's Hospital, Shanghai, China ${ }^{5}$ Cardiovascular Division, Brigham and Women's Hospital, Boston, Massachusetts, USA

${ }^{6}$ Advanced Data Analytics, Enterprise Information Systems, Cedars-Sinai Medical Center, Los Angeles, California, USA

${ }^{7}$ Department of Public Health Sciences and Comprehensive Cancer Center, University of California, Davis, Davis, California, USA

${ }^{8}$ Department of Medicine, Cedars-Sinai Medical Center, Los Angeles, California, USA

${ }^{9}$ Cedars-Sinai Cancer, Cedars-Sinai Medical Center, Los Angeles, California, USA

Acknowledgements We are grateful to all the front-line healthcare workers in our health system who continue to be dedicated to delivering the highest quality care for all patients.

Contributors JE, SC and JCF conceived and designed the study and participated in data collection, analysis, and writing and revision of the manuscript. MD, HJ, EHK, AH, TTN and SH participated in the analysis and writing of the manuscript. BC, MW, $E L, N S, P B, J D, E P$ and TD participated in the analysis.

Funding This work was supported by NIH grants U54 CA260591 and K23HL153888, the F Widjaja Family Foundation, the Helmsley Charitable Trust, the Cedars-Sinai Medical Center and the Erika J Glazer Family Foundation.

Competing interests None declared.

Patient consent for publication Not required.

Ethics approval All study protocols were approved by the Cedars-Sinai Institutional Review Board, with requirement for consent waived for use of deidentified data.

Provenance and peer review Not commissioned; externally peer reviewed by Joseph Katz, University of Florida, USA.

Data availability statement Data are available upon reasonable request. Data that support the findings of this study are available from Cedars-Sinai Medical Center. Data are not publicly available due to content including information that could compromise research participant privacy/consent.

Supplemental material This content has been supplied by the author(s). It has not been vetted by BMJ Publishing Group Limited (BMJ) and may not have been peer-reviewed. Any opinions or recommendations discussed are solely those of the author(s) and are not endorsed by BMJ. BMJ disclaims all liability and responsibility arising from any reliance placed on the content. Where the content includes any translated material, BMJ does not warrant the accuracy and reliability of the translations (including but not limited to local regulations, clinical guidelines, terminology, drug names and drug dosages), and is not responsible for any error and/or omissions arising from translation and adaptation or otherwise.

Open access This is an open access article distributed in accordance with the Creative Commons Attribution Non Commercial (CC BY-NC 4.0) license, which permits others to distribute, remix, adapt, build upon this work non-commercially, and license their derivative works on different terms, provided the original work is properly cited, appropriate credit is given, any changes made indicated, and the use is non-commercial. See: http://creativecommons.org/licenses/by-nc/4.0/.

ORCID iD

Joseph E Ebinger http://orcid.org/0000-0002-0587-1572

\section{REFERENCES}

1 Godoy M, Wood D. What do coronavirus racial disparities look like state by state? National Public Radio [serial on the Internet], 2020. Available: https://www.npr.org/sections/health-shots/2020/05/30/ 
865413079 /what-do-coronavirusracial-disparities-look-like-statebystate [Accessed 15 Jul 2020].

2 Schroeder K, Fahey T, Ebrahim S. Interventions for improving adherence to treatment in patients with high blood pressure in ambulatory settings. Cochrane Database Syst Rev 2004;2:CD004804.

3 Mays JC, Newman A. Virus is twice as deadly for Black and Latino people than Whites in N.Y.C. New York Times [serial on the Internet], 2020. Available: https://www.nytimes.com/2020/04/08/nyregion/ coronavirus-racedeaths.html [Accessed $15 \mathrm{Jul}$ 2020].

4 Brunenberg DEM, Wetzels GEC, Nelemans PJ, et al. Cost effectiveness of an adherence-improving programme in hypertensive patients. Pharmacoeconomics 2007;25:239-51.

5 Chapman RH, Ferrufino CP, Kowal SL, et al. The cost and effectiveness of adherence-improving interventions for antihypertensive and lipid-lowering drugs*. Int J Clin Pract 2010;64:169-81.

6 Health CDoP. COVID-19 race and ethnicity data [Internet] Sacramento (CA): CDPH, 2020. Available: https://www.cdph.ca. gov/Programs/CID/DCDC/Pages/COVID-19/Race-Ethnicity.aspx [Accessed 19 May 2020].

7 Krieger N, Testa C, Hanage WP, et al. Us racial and ethnic data for COVID-19 cases: still missing in action. Lancet 2020;396:e81.

8 Vann M, Kim SR. Minority groups at risk as states withhold, provide partial COVID-19 racial data. ABC News 2020;30.

9 Silverstein J. Democrats demand data on racial disparities in America's coronavirus response [Internet]. CBS News, 2020. Available: https://www.cbsnews.com/news/democrats-demanddata-on-racial-disparities-in-americascoronavirus-response/ [Accessed cited 2020 Mar 31].

10 Kabarriti R, Brodin NP, Maron Ml, et al. Association of race and ethnicity with comorbidities and survival among patients with COVID-19 at an urban medical center in New York. JAMA Netw Open 2020;3:e2019795.

11 Kaufman HW, Niles JK, Nash DB. Disparities in SARS-CoV-2 positivity rates: associations with race and ethnicity. Popul Health Manag 2021;24:20-6.

12 Webb Hooper M, Nápoles AM, Pérez-Stable EJ. COVID-19 and racial/ethnic disparities. JAMA 2020;323:2466-7.

13 Bonito AJ BC, Eicheldinger C, Carpenter L. Creation of new Race-Ethnicity codes and socioeconomic status (Ses) indicators for Medicare beneficiaries. final report, Sub-Task 2. Agency for Healthcare Research and Policy 2008.

14 van Walraven C, Austin PC, Jennings A, et al. A modification of the Elixhauser comorbidity measures into a point system for hospital death using administrative data. Med Care 2009;47:626-33.

15 Tong A, Baumgart A, Evangelidis N, et al. Core outcome measures for trials in people with coronavirus disease 2019: respiratory failure, multiorgan failure, shortness of breath, and recovery. Crit Care Med 2021:49:503-16.

16 Lesniak KT, Dubbert PM. Exercise and hypertension. Curr Opin Cardiol 2001;16:356-9.

17 Azar KMJ, Shen Z, Romanelli RJ, et al. Disparities in outcomes among COVID-19 patients in a large health care system in California. Health Aff 2020;39:1253-62.

18 Martinez DA, Hinson JS, Klein EY, et al. SARS-CoV-2 positivity rate for Latinos in the Baltimore-Washington, DC region. JAMA 2020;324:392-5.
19 Mukherjee V, Toth AT, Fenianos M, et al. Clinical outcomes in critically ill coronavirus disease 2019 patients: a unique New York City public hospital experience. Crit Care Explor 2020;2:e0188.

20 Price-Haywood EG, Burton J, Fort D, et al. Hospitalization and mortality among black patients and white patients with Covid-19. N Engl J Med 2020;382:2534-43.

21 Yancy CW. COVID-19 and African Americans. JAMA 2020;323:1891-2.

22 Selden TM, Berdahl TA. COVID-19 and racial/ethnic disparities in health risk, employment, and household composition. Health Aff 2020;39:1624-32.

23 Mahajan UV, Larkins-Pettigrew M. Racial demographics and COVID-19 confirmed cases and deaths: a correlational analysis of 2886 us counties. J Public Health 2020;42:445-7.

24 Niedzwiedz CL, O'Donnell CA, Jani BD, et al. Ethnic and socioeconomic differences in SARS-CoV-2 infection: prospective cohort study using UK Biobank. BMC Med 2020;18:160.

25 Raisi-Estabragh Z, McCracken C, Bethell MS, et al. Greater risk of severe COVID-19 in Black, Asian and Minority Ethnic populations is not explained by cardiometabolic, socioeconomic or behavioural factors, or by $25(\mathrm{OH})$-vitamin $\mathrm{D}$ status: study of 1326 cases from the UK Biobank. J Public Health 2020;42:451-60.

26 Asch DA, Sheils NE, Islam MN, et al. Variation in US hospital mortality rates for patients admitted with COVID-19 during the first 6 months of the pandemic. JAMA Intern Med 2020. doi:10.1001/ jamainternmed.2020.8193. [Epub ahead of print: 22 Dec 2020].

27 Beigel JH, Tomashek KM, Dodd LE, et al. Remdesivir for the treatment of Covid-19 - final report. New England Journal of Medicine 2020;383:1813-26.

28 Kalil AC, Patterson TF, Mehta AK, et al. Baricitinib plus Remdesivir for hospitalized adults with Covid-19. N Engl J Med 2021;384:795-807.

29 Weinreich DM, Sivapalasingam S, Norton T, et al. REGN-COV2, a neutralizing antibody cocktail, in outpatients with Covid-19. N Engl J Med 2021;384:238-51.

30 Arcaya MC, Figueroa JF. Emerging trends could exacerbate health inequities in the United States. Health Aff 2017;36:992-8.

31 Deaton A. Policy implications of the gradient of health and wealth. Health Aff 2002;21:13-30.

32 Deaton A. The great escape : health, wealth, and the origins of inequality. Princeton University Press: Princeton, 2013.

33 DBG T, Shah A, Doubeni CA. The disproportionate impact of COVID-19 on racial and ethnic minorities in the United States. Clinical Infectious Diseases 2020.

34 Allard L, Ouedraogo E, Molleville J, et al. Malnutrition: percentage and association with prognosis in patients hospitalized for coronavirus disease 2019. Nutrients 2020;12. doi:10.3390/ nu12123679. [Epub ahead of print: 28 Nov 2020].

35 Radujkovic A, Hippchen T, Tiwari-Heckler S, et al. Vitamin $D$ deficiency and outcome of COVID-19 patients. Nutrients 2020;12:2757.

36 Ogedegbe G, Ravenell J, Adhikari S, et al. Assessment of racial/ ethnic disparities in hospitalization and mortality in patients with COVID-19 in New York City. JAMA Netw Open 2020;3:e2026881.

37 Li K, Shakib S. How privilege shaped the COVID-19 pandemic. Contexts 2020;19:74-7.

38 Fujimura JH, Rajagopalan R. Different differences: the use of 'genetic ancestry' versus race in biomedical human genetic research. Soc Stud Sci 2011;41:5-30. 